\title{
On the Dirichlet problem for unbounded boundary functions
}

\author{
By Zenjiro Kuramochi and Yukio Nagasaka \\ (Received April 26, 1979)
}

1. We study the boundary behavior of the Dirichlet solution for an unbounded boundary function in this paper. We treat the Dirichlet problem by the Perron-Wiener-Brelot method. Let $G$ be a bounded open set in the complex plane and let $f(b)$ be an extended real-valued function defined on its boundary $\partial G$. The upper class $U_{f}^{f}$ for $f$ is given by

$$
\begin{aligned}
U_{f}^{G}= & \{s ; \text { superharmonic, bounded below on } G, \\
& \frac{\left.\lim _{z \rightarrow b} s(z) \geqq f(b) \text { for all } b \in \partial G\right\}}{}
\end{aligned}
$$

and set $\bar{H}_{f}^{\theta}(z)=\inf \left\{s(z) ; s \in U_{f}^{\theta}\right\}$ and $\underline{H}_{f}^{\theta}(z)=-\bar{H}_{-f}^{\theta}(z)$ for $z \in G$. If $\bar{H}_{f}^{\theta}=$ $\underline{H}_{f}^{q}$ and both harmonic, then $f$ is called to be resolutive and $H_{f}^{q}=\bar{H}_{f}^{\theta}=$ $\underline{H}_{f}^{G}$ is called the Dirichlet solution for $f$. Any bounded continuous function on $\partial G$ is resolutive. A point $b \in \partial G$ is called a regular boundary point if

$$
\lim _{z \rightarrow b} H_{g}^{G}(z)=g(b)
$$

for every bounded continuous function $g$ on $\partial G$. Otherwise $b$ is an irregular boundary point. The regularity is a local character, that is, if $b$ is a regular boundary point for $G$ and $G_{0}$ is an open subset of $G$ for which $b$ is also a boundary point, then $b$ is regular for $G_{0}$.

Let $b \in \partial G$ be a regular boundary point. By M. Brelot's example [1], we know that (1) does not always hold for any unbounded function, even if it is continuous and resolutive. In this paper, we give a sufficient condition for (1) to hold. Our result is the following:

THEOREm. Let $f$ be an extended real-valued continuous and resolutive function on $\partial G$. Suppose $b_{0} \in \partial G$ is a regular boundary point. If there is $a$ disk $B\left(b_{0}, r_{0}\right)=\left\{z ;\left|z-b_{0}\right|<r_{0}\right\}$ such that the Dirichlet integral $D_{G \cap B\left(b_{0}, r_{0}\right)}$ $\left(H_{f}^{G}\right)$ of $H_{f}^{G}$ on $G \cap B\left(b_{0}, r_{0}\right)$ is finite, then $\lim _{z \rightarrow b_{0}} H_{f}^{G}(z)=f\left(b_{0}\right)$.

For another sufficient condition, we refer to W. Ogawa [3].

2. The proof of the theorem. We refer to the monograph of Constantinescu-Cornea [2] for the definition and the properties of Dirichlet fun- 
ctions. We denote by $E$ the set of all irregular boundary points of $\partial G$.

First assume that $f \geqq 0$ on $\partial G$. For every positive integer $m$, define $f_{m}(b)=\min (f(b), m)$ and $H_{m}=H_{f_{m}}^{\theta}$. Since $f_{m}$ is bounded continuous on $\partial G$,

$$
\lim _{z \rightarrow b} H_{m}(z)=f_{m}(b) \quad \text { for every } b \in \partial G-E .
$$

Since $f_{m} \uparrow f, H_{m} \uparrow H_{f}^{g}$ on $G$. Since $\lim _{z \rightarrow b_{0}} H_{f}^{g}(z) \geqq \lim _{z \rightarrow b_{0}} H_{m}(z)=f_{m}\left(b_{0}\right)$ for every $m$, we have

$$
\lim _{z \rightarrow b_{0}} H_{f}^{G}(\boldsymbol{z}) \geqq f\left(b_{0}\right) .
$$

From this it follows that if $f\left(b_{0}\right)=+\infty, \lim _{z \rightarrow b_{0}} H_{f}^{G}(z)=+\infty$. Therefore we can assume $f\left(b_{0}\right)<+\infty$. Take an integer $m_{0}$ such that $f\left(b_{0}\right)<m_{0}$. Then there is a disk $B_{1}=B\left(b_{0}, r_{1}\right)$ such that $0<r_{1}<r_{0}$ and $f(b)<m_{0}$ for every $b \in \partial G \cap$ $B_{1}$. For every $n$ and $m$ such that $n>m \geqq m_{0}$, define

$$
S_{n, m}=H_{n}-H_{m} \text {. }
$$

Since $f_{n}(b)=f_{m}(b)=f(b)$ for every $b \in \partial G \cap B_{1}$, it follows from (2) that

$$
\lim _{z \rightarrow b} S_{n, m}(z)=0 \quad \text { for every } b \in(\partial G-E) \cap B_{1} .
$$

We denote by $I$ the component of $\partial G$ which contains $b_{0}$.

Case 1. The case where $I$ is a single point $b_{0}$. In this case, there is a Jordan region $D \subset B_{1}$ such that $b_{0} \in D$ and $\partial D \subset G$. Define a function $f_{0}$ on $\partial(G \cap D)$ as follows :

$$
f_{0}(b)= \begin{cases}H_{f}^{G}(b) & \text { if } b \in \partial D, \\ 0 & \text { if } b \in \partial G \cap D .\end{cases}
$$

Then $f_{0}$ is bounded continuous and so resolutive. Consider $H_{f_{0}}^{G \cap D}-S_{n, m}$, which is bounded harmonic on $G \cap D$. Since every point of $\partial D$ is regular for $G \cap D$,

$$
\lim _{z \rightarrow b}\left(H_{f_{0}}^{G \cap D}(z)-S_{n, m}(z)\right)=H_{f}^{q}(b)-\left(H_{n}(b)-H_{m}(b)\right) \geqq 0
$$

for every $b \in \partial D$, and by $(3), \lim _{z \rightarrow b}\left(H_{f_{0}}^{(\sigma \cap D}(\boldsymbol{z})-S_{n, m}(\boldsymbol{z})\right)=0$ for every $b \in(\partial G-$ $E) \cap D$. Since $E$ is a polar set and $\partial(G \cap D)-E=\partial D \cup((\partial G-E) \cap D)$, this shows that

$$
S_{n, m}(z) \leqq H_{f_{0}}^{G \cap D}(z) \quad \text { on } G \cap D .
$$

Letting $n \rightarrow \infty$, we obtain $H_{f_{0}}^{G \cap D}(z) \geqq H_{f}^{G}(z)-H_{m}(z) \geqq 0$ on $G \cap D$. Since $b_{0}$ is regular for $G \cap D$ and $G, \lim _{z \rightarrow b_{0}} H_{f_{0}}^{G \cap D}(z)=0$ and $\lim _{z \rightarrow b_{0}} H_{m}(z)=f_{m}\left(b_{0}\right)=f\left(b_{0}\right)$. Thus we have $\lim _{\theta \cap D \ni z \rightarrow b_{0}} H_{f}^{\theta}(\boldsymbol{z})=\lim _{\theta \ni z \rightarrow b_{0}} H_{f}^{\theta}(\boldsymbol{z})=f\left(b_{0}\right)$. 
Case 2. The case where $I$ is a continuum. Set $\alpha=D_{G \cap B_{1}}\left(H_{f}^{g}\right)<+\infty$. Let $f^{\prime}$ be a boundary function on $\partial\left(G \cap B_{1}\right)$ which is equal to $H_{f}^{\theta}$ on $\partial\left(G \cap B_{1}\right)$ $\cap G$ and to $f$ on $\partial\left(G \cap B_{1}\right) \cap \partial G$. Then $f^{\prime}$ is resolutive and $H_{f^{\prime}}^{G \cap B_{1}}=H_{f}^{\theta}$ on $G \cap B_{1}$. Set $H_{m}^{\prime}=H_{\min \left(f^{\prime}, m\right)}^{G \cap B_{1}}$ for every positive integer $m$. Then $H_{m}^{\prime} \uparrow H_{f}^{\theta}$ on $G \cap B_{1}$ as $m \rightarrow \infty$ and

$$
\lim _{z \rightarrow b} H_{m}^{\prime}(z)=\min \left(f^{\prime}(b), m\right)=f(b)
$$

for every $b \in(\partial G-E) \cap B_{1}$ and every $m \geqq m_{0}$. By the harmonic decomposition of Royden (cf. e.g. Satz 7.6 in [2]) we see that

$$
D_{G \cap B_{1}}\left(H_{m}^{\prime}\right) \leqq D_{G \cap B_{1}}\left(\min \left(H_{f^{\prime}}^{G \cap B_{1}}, m\right)\right) \leqq D_{G \cap B_{1}}\left(H_{f^{\prime}}^{G \cap B_{1}}\right)=\alpha .
$$

For every $n$ and $m$ such that $n>m \geqq m_{0}$, define $S_{n, m}^{\prime}=H_{n}^{\prime}-H_{m}^{\prime}$. Then

$$
\lim _{z \rightarrow b} S_{n, m}^{\prime}(z)=0 \quad \text { for every } b \in(\partial G-E) \cap B_{1}
$$

by (4) and $D_{G \cap B_{1}}\left(S_{n, m}^{\prime}\right) \leqq 2 D_{G \cap B_{1}}\left(H_{n}^{\prime}\right)+2 D_{G \cap B_{1}}\left(H_{m}^{\prime}\right) \leqq 4 \alpha$.

Let $G_{n, m}=\left\{z \in G \cap B_{1} ; S_{n, m}(z)>1\right\}$. Then $\partial G_{n, m} \cap B_{1}=\left(\partial G_{n, m} \cap B_{1} \cap G\right) \cup$ $\left(\partial G_{n, m} \cap B_{1} \cap \partial G\right)$, where $\partial G_{n, m} \cap B_{1} \cap G$ is analytic and $E_{n, m}=\partial G_{n, m} \cap B_{1} \cap \partial G$ is closed relative to $B_{1}$ and has capacity zero by $E_{n, m} \subset E$. Define a function $T_{n, m}$ on $B_{1}$ as follows:

$$
T_{n, m}(z)= \begin{cases}S_{n, m}^{\prime}(z)-1 & \text { if } z \in G_{n, m} \\ 0 & \text { if } z \in B_{1}-G_{n, m}\end{cases}
$$

Then we see that $T_{n, m}$ is continuous on $B_{1}-E_{n, m}$, a Tonelli function on $B_{1}$ and

$$
D_{B_{1}}\left(T_{n, m}\right) \leqq D_{G \cap B_{1}}\left(S_{n, m}^{\prime}\right) \leqq 4 \alpha,
$$

that is; $T_{n, m}$ is a Dirichlet function on $B_{1}$. Take $r_{2}, r_{3}$ and $r_{4}$ such that $r_{1}>r_{2}>r_{3}>r_{4}>0$ and set $B_{i}=B\left(b_{0}, r_{i}\right), i=2,3,4$. Since $E_{n, m}$ is a polar set, we may take $r_{2}$ such that $\partial B_{2} \cap\left(\cup_{n, m} E_{n, m}\right)=\phi$. Let $I_{0}$ be the component of $I \cap \bar{B}_{4}$ which contains $b_{0}$. Then $I_{0}$ is also a continuum and $T_{n, m}=0$ on $I_{0}$. Let $w_{i}(z)(i=2,3)$ be the harmonic measure of $\partial B_{i}$ with respect to $B_{i}-I_{0}$. Then $w_{i}$ can be continuously extend to $\bar{B}_{i}$ such that

$$
w_{i}=0 \text { on } I_{0} \text { and } w_{i}=1 \text { on } \partial B_{i} .
$$

In $B_{2}-I_{0}$, there is only one component $J_{0}$ such that $\partial J_{0} \supset \partial B_{2}$ and the other components $J_{i}(i \geqq 1)$ satisfy $\partial J_{i} \subset I_{0}$. Let $g_{n, m}$ be the restriction of $T_{n, m}$ to $\partial\left(B_{2}-I_{0}\right)=\partial B_{2} \cup I_{0}$. Then $g_{n, m}$ is bounded continuous on $\partial B_{2} \cup I_{0}$. Let $U_{n, m}$ be the Dirichlet solution corresponding to $B_{2}-I_{0}$ and the boundary function $g_{n, m}$. Then we have 


$$
D_{B_{2}-I_{0}}\left(U_{n, m}\right) \leqq D_{B_{2}}\left(T_{n, m}\right) \leqq 4 \alpha
$$

by the Dirichlet principle (p. 155 in [2]). Let $\beta_{n, m}=\min _{z \in \partial B_{3}} U_{n, m}(z) \geqq 0$. Then $D_{B_{3}-I_{0}}\left(U_{n, m}\right) \geqq D_{B_{3}-I_{0}}\left(\min \left(U_{n, m}, \beta_{n, m}\right)\right)$. Since $\min \left(U_{n, m}, \beta_{n, m}\right)=\beta_{n, m} w_{3}$ on $\partial B_{3}$ $\cup I_{0}, D_{B_{3}-I_{0}}\left(\min \left(U_{n, m}, \beta_{n, m}\right)\right) \geqq \beta_{n, m}^{2} D_{B_{3}-I_{0}}\left(w_{3}\right)$ by the Dirichlet principle. Hence we have

$$
D_{B_{3}-I_{0}}\left(U_{n, m}\right) \geqq \beta_{n, m}^{2} D_{B_{3}-I_{0}}\left(w_{3}\right) .
$$

Consider $U_{n, m}+w_{2}-S_{n, m}^{\prime}$ on $G \cap B_{2}$. By

$$
\begin{aligned}
& \lim _{z \rightarrow b}\left(U_{n, m}(z)+w_{2}(z)\right)=g_{n, m}(b)+1=S_{n, m}^{\prime}(b) \quad \text { if } b \in \partial B_{2} \cap G_{n, m}, \\
& \lim _{z \rightarrow b} w_{2}(z)=1 \geqq S_{n, m}^{\prime}(b) \quad \text { if } b \in \partial B_{2} \cap\left(G-G_{n, m}\right)
\end{aligned}
$$

and (5), we have $\lim _{z \rightarrow b}\left(U_{n, m}(z)+w_{2}(z)-S_{n, m}^{\prime}(z)\right) \geqq 0$ for every $b \in \partial\left(G \cap B_{2}\right)-E$. This shows that

$$
S_{n, m}^{\prime}(z) \leqq U_{n, m}(z)+w_{2}(z) \quad \text { on } G \cap B_{2} .
$$

Consider $\left\{U_{n, m}\right\}_{n, m}$ on $J_{0}$ (Note $U_{n, m}=0$ on $J_{i}(i \geqq 1)$ ). Suppose $\sup _{n, m} U_{n, m}\left(z_{0}\right)$ $=+\infty$ for some point $z_{0} \in J_{0}$. Then $\sup _{n, m} \min _{z \in B} U_{n, m}(z)=+\infty$ by Harnack theorem. Since $D_{J_{0}}\left(U_{n, m}\right) \geqq\left(\min _{z \in \partial B_{3}} U_{n, m}(z)\right)^{2} D_{J_{0} \cap B_{3}}\left(w_{3}\right)$ by (8) this implies $\sup _{n, m}$ $D_{J_{0}}\left(U_{n, m}\right)=+\infty$. But this contradicts (7). Hence we have $\sup _{n, m} U_{n, m}\left(z_{0}\right)<$ $+\infty$. This shows that $\sup _{n, m} \max _{z \in \partial B_{3}} U_{n, m}(z)=\gamma<+\infty$ by Harnack theorem. Since $\lim _{z \rightarrow b} U_{n, m}(z)=0$ for every $b \in I_{0}$, we have

$$
U_{n, m}(z) \leqq \gamma w_{3}(z) \quad \text { on } B_{3}-I_{0} .
$$

Hence $0 \leqq S_{n, m}^{\prime}(z) \leqq \gamma w_{3}(z)+w_{2}(z)$ on $G \cap B_{3}$ by (9). Letting $n \rightarrow \infty, 0 \leqq$ $H_{f^{\prime}}^{G \cap B_{1}}(z)-H_{m}^{\prime}(z) \leqq \gamma w_{3}(z)+w_{1}(z)$. Consequently we conclude from (4) and (6) $\lim _{z \rightarrow b_{0}} H_{f}^{G}(z)=\lim _{z \rightarrow b_{0}} H_{f}^{G \cap B_{1}}(z)=f\left(b_{0}\right)$.

Finally we remove the assumption that $f \geqq 0$ on $\partial G$. Since $f^{\prime}$ is resolutive, $g(b)=\max \left(f^{\prime}(b), 0\right)$ and $h(b)=\max \left(-f^{\prime}(b), 0\right)$ are resolutive and $H_{f^{\prime}}^{G \cap B_{1}}$ $=H_{g}^{G \cap B_{1}}-H_{h}^{G \cap B_{1}}$. Since $D_{G \cap B_{1}}\left(H_{f^{\prime}}^{G \cap B_{1}}\right)<+\infty$, we see that $D_{G \cap B_{1}}\left(H_{g}^{G \cap B_{1}}\right)<+\infty$ and $D_{G \cap B_{1}}\left(H_{h}^{G \cap B_{1}}\right)<+\infty$. From the above reasoning it follows that $\lim H_{g}^{G \cap B_{1}}$

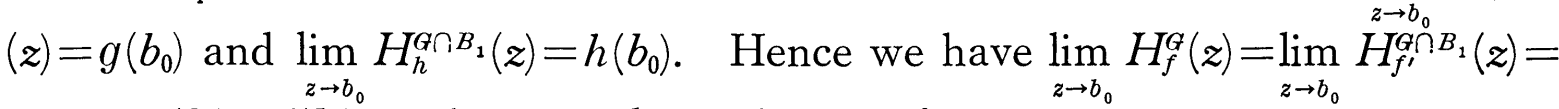
$g\left(b_{0}\right)-h\left(b_{0}\right)=f\left(b_{0}\right)^{\circ}$. This completes the proof. 


\section{References}

[1] M. BRelot: Sur la mesure harmonique et le probléme de Dirichlet, Bull. Sci. Math., (2) 69, 153-156 (1945).

[2] C. Constantinescu and A. CoRnea: Ideale Ränder Riemannscher Flächen, Springer (1963).

[3] W. OGAWA: Boundary behavior of Dirichlet solutions at regular boundary points, Hokkaido Math. J. (to appear).

Department of Mathematics

Hokkaido University 